\title{
AMBIENTES DOMÉSTICOS E QUEDAS ENTRE IDOSOS EM TEMPOS DE PANDEMIA DO COVID-19
}

\author{
Tuíra Oliveira Maia/UFPE \\ Laura Bezerra Martins/UFPE
}

\begin{abstract}
RESUMO
Introdução. O distanciamento social estabelecido pela pandemia de COVID-19 provocou uma maior permanência de idosos em seus ambientes domiciliares, o que resultou em uma maior exposição aos fatores de riscos ambientais. Objetivo: Identificar as características ambientais associadas às quedas ocorridas entre idosos no período de pandemia. Materiais e métodos: Estudo de caso realizado na enfermaria de um hospital público de Pernambuco através da avaliação de 28 idosos por meio do questionário Older Americans Resources and Services (OARS) e entrevista semiestruturada. Resultados: Os fatores ambientais associados às quedas que os participantes mais descreveram foram piso escorregadio, piso desnivelado; falta de equipamentos de apoio e móveis inadequados. Conclusão: Todos os episódios de quedas ocorridos entre idosos no período da pandemia estavam associados a um fator de risco ambiental, por isso, mais estratégias intersetoriais devem ser estudadas para reduzir a incidência destes riscos nas residências desta população.
\end{abstract}

Palavras-chave: Ambiente doméstico; COVID-19; Acidentes; Quedas; Idosos.

\section{Introdução}

Todos os anos, 30 milhões de pessoas da União Europeia com 60 anos ou mais são feridas por acidentes domésticos ou de lazer, sendo as quedas, a principal causa dessas lesões fatais entre os idosos (EUROPEAN ASSOCIATION FOR INJURY PREVENTION AND SAFETY PROMOTION, 2017). No Brasil, as quedas são consideradas problemas de saúde pública devido à alta demanda de internações (MASCARENHAS et al., 2011). De acordo com dados da Universidade Federal de São Paulo (USP), 13\% de idosos caem de forma recorrente, número que chegou a $30 \%$ na pandemia.

A pandemia foi decretada em 2020 pela Organização Mundial de Saúde (OMS) devido à disseminação acelerada do novo coronavírus (OPAS, 2020). A alta transmissibilidade da COVID-19 levou a adoção da estratégia de distanciamento e isolamento social. Essa medida fez com que os idosos passassem mais tempo dentro das suas residências, e consequentemente, mais tempo expostos a fatores de riscos ambientais, muitas vezes, existentes nos domicílios (DE LIMA et al.,2020).

Deste modo, apesar de todo avanço tecnológico, os acidentes domésticos ainda estão presentes nos domicílios de idosos devido à tendência dessa população em realizar adaptações ambientais 
inadequadas de mobiliários e ambientes, que não são apropriadas para as suas condições físicas atuais, assim como, a adoção de medidas preventivas, na maioria das vezes, apenas posteriormente a ocorrência dos acidentes (MARTINS et al., 2016).

Logo, diante do panorama atual, é essencial oferecer um ambiente seguro para manutenção da saúde dos idosos. Assim, objetivo do estudo é identificar o perfil ambiental de acidentes ocorridos entre idosos no período de pandemia.

\section{Materiais e métodos}

Trata-se de um estudo de caso realizado no período de junho a julho de 2021 , na enfermaria de traumatologia do Hospital Dom Helder Câmara, no Cabo de Santo Agostinho, Pernambuco. Foram avaliados 29 idosos através de uma amostra não probabilística intencional, na qual, foram incluídos todos os idosos (considerando idade igual ou maior que 60 anos), que permanecerem internados durante o período de coleta estabelecido, que aceitarem participar da pesquisa e estiverem com estabilidade clínica.

Para a realização do estudo de caso, o projeto de pesquisa foi submetido ao Comitê de Ética e Pesquisa da Universidade Federal de Pernambuco e aprovado com o CAAE: 44664021.1.0000.5208. Os participantes do estudo e/ou responsáveis foram devidamente esclarecidos sobre os objetivos da pesquisa e, só foram incluídos no estudo após a assinatura do Termo de Consentimento Livre e Esclarecido.

Para a coleta dos dados foi utilizado o questionário Older Americans Resources and Services (OARS) desenvolvido pela Duke University, em 1978. O OARS é um instrumento com várias dimensões para avaliar o estado funcional dos idosos, que foi validado e adaptado para realidade brasileira por Ramos (1987). Será utilizado nesta pesquisa parte do instrumento OARS, contendo apenas 9 perguntas. Ele é composto por dois itens: Identificação: sexo, idade, cor, estado civil, tempo e local de residência; Perfil social do idoso: nível de escolaridade, situação socioeconômica, atividade profissional. Foi realizada também uma entrevista semiestruturada para identificar as características das quedas, que não foram obtidas nos prontuários.

\section{Resultados}

Dos 28 idosos avaliados, a maioria era do sexo feminino, viúvos, aposentados e que atualmente, não exercem nenhuma atividade profissional. A média de idade foi de 73,1 anos, $60,72 \%$ moravam na 
zona rural, grande parte mora por mais de 10 anos na residência onde sofreu o acidente. A tabela 1 mostra mais informações do perfil dos idosos avaliados.

Tabela 1. Perfil social do idoso participante da pesquisa

\begin{tabular}{|c|c|c|c|}
\hline Variável & Média & $n$ & $\%$ \\
\hline Idade (anos) & 73,1 & 28 & - \\
\hline \multicolumn{4}{|l|}{ Sexo } \\
\hline Feminino & - & 21 & 75 \\
\hline Masculino & - & 7 & 25 \\
\hline \multicolumn{4}{|l|}{ Escolaridade } \\
\hline Analfabeto & & 4 & 14,28 \\
\hline Sabe ler e escrever & & 3 & 10,71 \\
\hline 1 a 8 anos & & 15 & 53,58 \\
\hline$>9$ anos & & 4 & 14,28 \\
\hline Não sabe & & 2 & 7,15 \\
\hline \multicolumn{4}{|l|}{ Estado Conjugal } \\
\hline Solteiro & & 4 & 14,27 \\
\hline Casado & & 7 & 25 \\
\hline Viúvo & & 15 & 53,58 \\
\hline Divorciado & & 2 & 7,15 \\
\hline \multicolumn{4}{|l|}{ Tempo de moradia } \\
\hline$<10$ anos & - & 3 & 10,71 \\
\hline$>10$ anos & - & 25 & 89,28 \\
\hline \multicolumn{4}{|l|}{ Área de moradia } \\
\hline Urbana & - & 11 & 39,28 \\
\hline Rural & - & 17 & 60,72 \\
\hline
\end{tabular}

$\mathrm{Na}$ avaliação das características ambientais associadas às quedas percebe-se que os locais das quedas variaram entre cozinha, banheiro, área de serviço, corredor, sobretudo, quarto, sala e 
quintal. Os motivos relatados mais comuns foram: Piso escorregadio/piso desnivelado; falta de equipamentos de apoio, móveis inadequados e objetos espalhados. Além disso, 18 idosos $(64,28 \%)$ relatam possuir móveis considerados de risco para acidentes. Dez $(35,71 \%)$ das quedas ocorridas estavam associadas ao ato de levantar de algum móvel (cama ou cadeira) e 12 (42,85\%) foram escorregões em superfícies molhadas.

Os participantes também descreveram ter medo de sofrer acidentes principalmente no banheiro. Dentre as medidas utilizadas para reduzir o risco da queda descreveram a utilização de dispositivos auxiliares como andadores e bengalas, uso de sapato antiderrapante e apenas $7,14 \%$ dos idosos possuíam equipamentos de apoio na sua residência. A maioria dos participantes teve mais de um episódio de queda em sua residência. A tabela 2 demonstra todos os fatores de risco domiciliares citados pelos participantes

\section{Presente}

Fatores de risco para quedas

Piso escorregadio/desnivelado

Falta de equipamentos de

apoio

Móveis inadequados

Objetos espalhados

Configuração do ambiente

inadequada

\section{$\mathrm{N}$}

20

5

9

1

2
$\%$

71,42

17,85

32,14

3,57

7,14

\section{Discussão}

Os fatores de risco ambientais que foram encontrados em todos os episódios de quedas relatados pelos participantes do estudo ocorridas no período de pandemia. Um estudo de 2012 demonstrou que os fatores de risco ambientais são significantes preditores de quedas, visto que, o risco de cair é $1,39 \%$ maior entre idosos que residem em casas com pisos escorregadios (SOPHONRATANAPOKIN et al., 2012). 
No presente estudo, fatores como piso escorregadio/piso desnivelado, falta de equipamentos de apoio e móveis inadequados foram os mais relatados, entretanto, sabe-se que outros fatores podem estar atuando sinergicamente com essas características, como: má iluminação e obstáculos no interior da casa (NETO et al., 2018). Contudo, o risco ambiental parece não ser suficiente para a ocorrência de quedas, mas sim a interação entre as condições dos idosos e o grau de exposição aos fatores de risco ambiental (OLIVEIRA et al., 2014).

No entanto, apesar de ser evidente a necessidade de mudanças ambientais para reduzir a ocorrência de quedas, não deve-se ignorar as questões sociais limitantes a qual a maioria dos idosos vivem. Portanto, é necessário estabelecer estratégias intersetoriais que busquem alternativas e soluções que visem reduzir os dados gerados pelas iniquidades e desigualdades do país (GUEDES et al., 2017).

\section{Conclusão}

Após a realização do estudo pode-se concluir que todos os acidentes que ocorreram com idosos dentro de seus domicílios no período de pandemia estão associados a componentes ambientais, sobretudo pela utilização de móveis inadequados e pisos impróprios para esse tipo de população independente da área da residência ser rural ou urbana. Deste modo, percebe-se a necessidade de adoção de medidas intersetoriais no ambiente para fornecer um ambiente mais seguro e ergonômico para os idosos contribuindo para a redução de acidentes com repercussões físicas, emocionais, sociais e para os serviços de saúde.

\section{Referências}

DE LIMA, Morgana Cristina; DA SILVA SIMPLICIO, Mônica Rita; HOLANDA, Elaine Cristina dos Santos. Educação em saúde sobre fatores de risco na ocorrência de quedas em idosos em tempos de pandemia do COVID-19: Relato de experiência. Braz. J. of Dev., v. 6, n. 8, p. 58825-58830, 2020. DOI:10.34117/bjdv6n8-334.

EUROPEAN ASSOCIATION FOR INJURY PREVENTION AND SAFETY PROMOTION. Injuries in the European Union, Report on injury statistics 2008- 2010, Amsterdam, 2013. Disponível em: https://ec.europa.eu/health//sites/health/files/data_collection/docs/idb_report_2013_en.p df.

Guedes MBOG, Lima KC, Caldas CP, Veras RP. Apoio social e o cuidado integral à saúde do idoso. Physis [Internet]. v. 27, n.4, p. 1185-1204, 2017.

MARTINS, L.; BARKOKÉBAS, B.; BAPTISTA, J.; AREZES, P. Domestic Safety and Accidents Risk Perception by Active Elderly. In: Arezes P. (eds) Advances in Safety Management and Human Factors. Advances in Intelligent Systems and Computing, vol 491. Springer, Cham, 2016.

MASCARENHAS, M.D.M; MONTEIRO, R.A.; SÁ, N.N.B.; GONZAGA, L.A.A.; NEVES, A.C.M.; SILVA, M.M.A.; MALTA, D.C. Epidemiologia das causas externas no Brasil: morbidade por acidentes e violências In: Ministério da Saúde (BR). Saúde Brasil 2010: uma análise da situação de saúde e de 
evidências selecionadas de impacto de ações de vigilância em saúde. Brasília: Ministério daSaúde; 2011. p. $203-24$.

Oliveira, Adriana Sarmento de et al. Fatores ambientais e risco de quedas em idosos: revisão sistemática. Revista Brasileira de Geriatria e Gerontologia [online]. 2014, v. 17, n. 3 [Acessado 22 Julho 2021] , pp. 637-645. Disponível em: <https://doi.org/10.1590/1809-9823.2014.13087>.

OPAS- Folha informativa - COVID-19 (doença causada pelo novo coronavírus).Disponível em: https://www.paho.org/bra/index.php?option=com content\&view=article\&id=6101:covid19\&ltemi $\mathrm{d}=875$. Acesso em: 21 jul. 2020.

Sophonratanapokin B, Sawangdee Y, Soonthorndhada K. Effect of the living environment on falls among the elderly in Thailand. Southeast Asian J Trop Med Public Healt 2012;43(6):1537-47. 